\title{
Host-parasite interface of Filicollis anatis (Palaeacanthocephala) in domestic ducks
}

\author{
Horst Taraschewski ${ }^{1}$, Ulrich Hofmann ${ }^{2}$ \\ ${ }^{1}$ Institut für Spezielle Zoologie und Parasitologie, Ruhr-Universität-Bochum, W-4630 Bochum, Germany \\ ${ }^{2}$ Flurstr.6a, O-7261 Calbitz, Germany
}

\begin{abstract}
The host-parasite interface of Filicollis anatis (Palaeacanthocephala) in domestic ducks was investigated by electron microscopy. The tissue alterations caused by mature female worms were more conspicuous than those around the presoma of male worms and immature females. The bulbus of mature females always projected into the ducks' peritoneal cavity. The host's inflammatory tissue was characterized by abundant heterophilic granulocytes and macrophage/epitheloid cells and the presence of macrophage giant cells. Its composition differed along the length of the mature female's presoma. At the bulbus and the anterior part of the neck a broad belt of degenerated reactionary tissue with accumulations of a wax-like substance and containing foreign bodies bordered the homogeneous surface coat of the presoma. Occasionally the tissue surrounding the bulbus seemed to become torn so that the worm and the peritoneal fluid were in direct contact. Results are discussed and compared with acanthocephalan infestations in fishes and mammals.
\end{abstract}

\section{INTRODUCTION}

The host-parasite interface of acanthocephalans in fish as well as in mammals has been investigated by electron microscopy (Taraschewski 1989a, b, Taraschewski et al. 1989, Zhão et al. 1990). Only descriptions using light microscopy, however, are available on the host-parasite interface of acanthocephalans in birds (Pflugfelder 1956, Moore \& Bell 1983). The aim of the present light and electron microscopical investigation is to describe the mechanical effects caused by acanthocephalans in domestic ducks, the host's response at the point of attachment and the appearance of that part of the parasite that is in close contact with the host's tissues. The palaeacanthocephalan Filicollis anatis is known to parasitize wild and domestic ducks throughout Europe (Soliman 1955, Styczynska 1958). In domestic ducks it has been found to cause mortality (Hofmann et al. 1989).

\section{MATERIALS AND METHODS}

Two domestic ducks were obtained from a private supplier and released at a brook which was known to harbour numerous isopods Asellus aquaticus infested with larval Filicollis anatis. The ducks foraged in the creek but also received kitchen wastes. After 2 mo the ducks were killed and their intestines were opened immediately in order to excise intestinal tissues.

Intestinal tissues with attached acanthocephalans were either used as squash preparations or were prepared for histology. Sections of up to $1 \mathrm{~cm}$ in diameter were fixed in Bouin's solution, embedded in paraffin, sectioned at 5 to $7 \mathrm{um}$ and stained with hematoxylineosin. Worms and surrounding tissues of up to $5 \mathrm{~mm}$ in diameter were also studied using semithin sections $(0.5 \mu \mathrm{m})$ prepared as follows: Samples were fixed in $5 \%$ glutaraldehyde buffered with $0.1 \mathrm{M}$ sodium cacodylate, $\mathrm{pH} 7.4$ at $4{ }^{\circ} \mathrm{C}$ for $24 \mathrm{~h}$. After fixation they were treated with $2 \% \mathrm{OSO}_{4}$, using the same buffer, and then dehydrated in graded ethanol, transferred to propylene oxide and embedded in araldite. Araldite sections were stained with methylene blue and Azur A.

For electron microscopy, intestinal samples were treated as described for semithin sections. Ultra-thin sections were stained with uranyl acetate and lead citrate. They were examined in a Zeiss EM9 S2.

\section{RESULTS}

The guts of the ducks contained 23 and 56 specimens, respectively, of Filicollis anatis. Most worms 


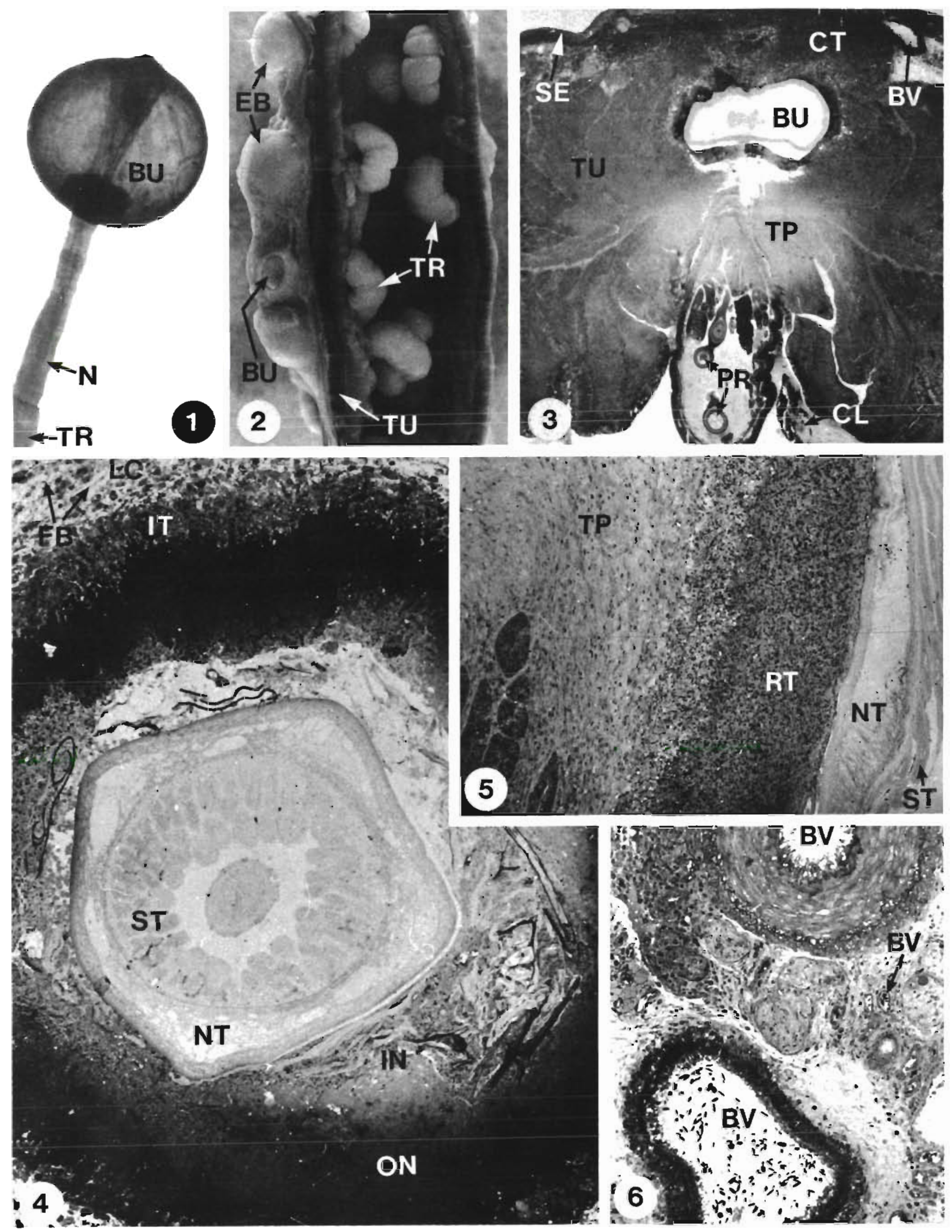


were mature females. The presoma of male worms, which measured up to about $8 \mathrm{~mm}$ in length, does not include a bulbus. It did not project into the peritoneal cavity of the ducks but was embedded in tissue of the intestinal wall. The presoma of female worms comprises a bulbus (Fig. 1). The depth of penetration of females depended on the size of the worms. Mature females with a length of 1 to $3 \mathrm{~cm}$ projected into the peritoneal cavity with their bulbus (Fig. 2) while the neck was surrounded by the tissue of the intestinal wall (Fig. 4). In contrast, females smaller than 8 to $10 \mathrm{~mm}$ in length had penetrated only the tunica muscularis of the intestinal wall (Fig. 3); thus the bulbus was not visible from the serosal surface of the intestinal wall. The presoma of all worms also comprises a proboscis. However, in female worms the proboscis is not well separated from the bulbus. In all sections the proboscis of male as well as of female worms usually was found half invaginated, so that it formed a cavity at the anterior tip of the worm.

Generally, the host's cellular reactions at the attachment site of small females and of male worms were less pronounced than those caused by mature females. A predominantly haemorrhagic reaction was noted around the presoma of these small worms. The tissue near the presoma consisted mainly of heterophilic granulocytes and erythrocytes. Macrophages occurred less frequently and fibroblasts and collagen fibres were also less frequently found or rare. Their number increased with increasing worm size. Plasma cells did not occur. The inflammatory tissue did not show a clear zonation as around the presoma of mature females. Fig. 3 shows an immature female worm which has been sectioned, the presoma of which is already surrounded by reaction tissue with different zones. Around the trunk of each male or immature female worm an exudate was found consisting mainly of necrotic leucocytes and fluids from necrotic tissue and from hypertrophic goblet cells. The exudate exuded from the lesions into the intestinal lumen (Fig. 3).

At the attachment site of mature females, the exudation of necrotic cells and mucus-like substance from the lesion was less than around male and immature female worms. The inflammatory tissue around the presoma of mature females was arranged in different zones. Around the mid neck and the posterior part of the neck infiltrating cells formed a solid mass (Fig. 5) which mainly consisted of macrophages (Fig. 7) partly converted into epitheloid cells. This tissue was interspersed with granulocytes, fibroblasts and collagen fibres (Fig. 7). In close proximity to the worm tegument it showed necrosis, but the necrotic band was narrow (Fig. 5).

At the anterior part of the neck and around the bulbus the host's inflammatory tissue consisted of different layers arranged around the worm. At close contact with the worm's tegument, a more or less broad necrotic band always existed (Fig. 4). The inner part of this necrotic band consisted of necrotic defense cells, mucus-like substance, drops or aggregations of markedly and of slightly osmiophilic matter (Figs. 9 \& 13), bacteria, filiform fungi, mineral grains and synthetic fibres (Fig. 4). The outer part of the necrotic zone was characterized by necrotic macrophage/epitheloid cell tissue (Fig. 8). These cells were closely connected by numerous protuberances of their outer surface. They contained many microfilaments and drops or vacuoles of slightly osmiophilic matter and large quantities of a highly osmiophilic, wax-like matter (Fig. 8). Often, the wax-like substance contributed more than $50 \%$ of a cell's cytoplasm. The nuclei and mitochondria of these cells often were lysed (Fig. 8).

Externally, the necrotic zone formed solid tissue with numerous macrophages interspersed with few heterophilic granulocytes, fibroblasts and collagen fibres (Fig. 7). The macrophages harboured intact nuclei and mitochondria and were rich in phagosomes and/or phagolysosomes (Fig. 7).

The solid macrophage band was bordered exteriorly by an outer loose mesh of entangled cells (Figs. 4 \& 6) comprising fibroblasts with surrounding collagen fibres, heterophilic granulocytes, macrophages, macrophage giant cells (Fig. 11) and a few lymphocytes. Hypertrophic blood vessels as well as blood capillaries

Figs. 1 to 6. Light micrographs of Filicollis anatis (Figs. 1 to 4 ) and surrounding tissue of the host's intestinal wall (Figs. 2 to 6). Fig. 1. Anterior half of a mature female worm. Note the long neck and the conspicuous bulbus. The small proboscis carrying many small hooks is partly retracted into the bulbus; $\times 10$. Fig. 2. Intestinal lumen showing several mature females. The trunks (metasoma) can be seen inside the intestine and the encapsulated bulbi at the outer side of the gut. One bulbus is not fully encapsulated; $\times 2$. Fig. 3. Semithin longitudinal section of an immature female worm protruding through the tissue of the intestinal wall. The bulbus does not yet project into the peritoneal cavity; $\times 40$. Fig. 4 . Semithin transverse section through the upper part of a mature female's neck and the surrounding tissue. Note the zonation of the reactive tissue; $\times 180$. Fig. 5. Semithin section through the anterior part of a mature female's neck and the solid unzonated tissue around; $\times 160$. Fig. 6 . Semithin section through the loose connective tissue at the outer part of the capsule enclosing the bulbus of a mature female; $\times 180$. BU: bulbus; BV: blood vessel; CL: cells and liquids discharged into the intestinal lumen; EB: encapsulated bulbus; FB: fibroblast; IN: inner belt of the necrotic zone; IT: intact macrophage tissue; LC: loose mesh of conective tissue; N: neck; NT: neck tegument; ON: outer belt of the necrotic zone; PR: proboscis receptaculum; RT: reactionary tissue; SE: serosa; ST: subtegumental musculature; TP: tunica propria; TR: trunk (metasoma); TU: tunica muscularis 

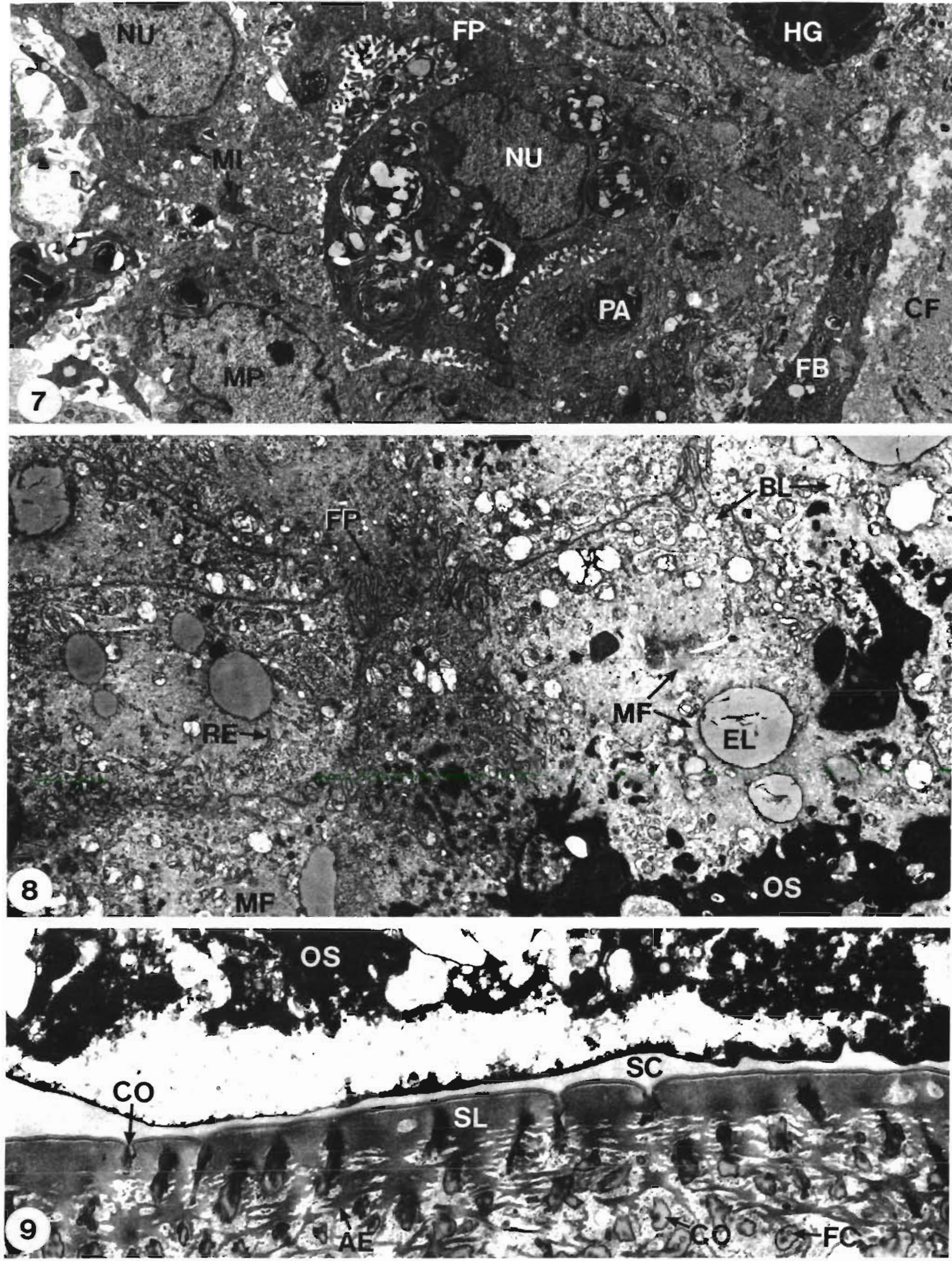
were contained in this tissue (Fig. 6), but blood cells (mainly erythrocytes and heterophilic granulocytes) were also found outside blood vessels in haemorrhagic foci (Fig. 10).

Occasionally, the worms seemed to have ruptured this tissue as well as the serosa. In such cases the bulbus was found in direct contact with the peritoneal fluid (Fig. 2).

Plasma cells (Fig. 12) occurred in all zones of the reactionary tissue around mature female worms, but they were never numerous in any of the zones.

Along their entire presoma all worms were covered with an electron-lucent, homogeneous substance (Fig. 9) which was separate from the wax-like, highly osmiophilic matter accumulating in the necrotic zone. However, it was continuous with the (probably liquid) substance inside the crypts of the tegument's outer limiting membrane (Fig. 9). Within the tegument's striped layer (Fig. 9) the crypts of the outer membrane were fused and thus formed large caverns which were interspersed with numerous supporting fibres. The presoma tegument with its homogeneous surface coat was rich in drops and vacuoles of different osmiophilic property. Especially the lemnisci (which are paired, sack-shaped outgrowths of the presoma tegument) contained many such structures in a generally osmiophilic matrix (Fig. 14).

\section{DISCUSSION}

The host-parasite interface of Filicollis anatis in domestic ducks seems to reflect on the one hand factors typical for palaeacanthocephalan infestations and on the other hand factors characterizing the cellular defense mechanisms of birds.

According to Taraschewski (1988a) there are perforating acanthocephalans, which penetrate deeply into or through the intestinal wall of their hosts, and non-perforating species with a superficial attachment to the mucosa. Among the perforating species such as the fish-parasitizing palaeacanthocephalan Acanthocephalus anguillae the depth of penetration seems to increase with the duration of infestation (Taraschewski 1989a). In the present study the exact age of the worms was not known, but it was assumed that the males, which usually are short-lived among acanthocephalans (Nickol 1985) and the small females are young specimens. The host's tissue response to the presoma of these worms can be considered an acute inflammatory reaction. At the point of attachment of large females, which were probably almost 2 mo old, the chronic infestation associated with the worms' deep penetration caused considerable tissue reaction.

The solid ring of macrophage/epitheloid cell tissue around the posterior part of the worms' neck, i.e. in the tunica propria of the host, seems to be essentially a stabilizing reaction, in order to protect the lesion and to prevent the influx of microorganisms from the intestinal lumen into the duct around the worm. The abundant foreign bodies in the necrotic zone around the neck were probably introduced into the duct during the attachment of the worm. Inside the body cavity, i.e. around the bulbus, the host does not need to build up a very solid reactionary tissue. The haemorrhagic foci within the outer loose mass of connective tissue seem to be due to occasional tearing of this tissue by the worm

A broad necrotic zone of host tissue around the anterior part of the presoma has also been described from perforating fish acanthocephalans (Taraschewski 1988a). The presoma of Filicollis anatis is covered with a homogeneous coat which is continuous with the contents of the crypts of the tegument's outer membrane. resembling the presoma surface of fish-parasitizing palaeacanthocephalans and eoacanthocephalans (Taraschewski 1989a, b). In fish as well as in ducks the homogeneous coat seems to contain lipids derived from the surrounding necrotic host tissue. It is likely that the homogeneous coat is a protective measure of the palaeacanthocephalan and the eoacanthocephalan presoma against the host's defence. The highly osmiophilic, wax-like substance accumulating at the presoma of $F$. anatis may consist of lipids with longchained fatty acids which are not incorporated into the (probably liquid) surface cover. In infestations of archiacanthocephalans in mammals such a cover does not exist (Taraschewski et al. 1989, Zhao et al. 1990).

Figs. 7 to 9. Transmission electron micrographs of duck host tissue near the upper part of the neck of a mature female Filicollis anatis and of the worm's tegument (Fig. 9). For location of tissues see Fig. 4. Fig. 7 . Intact macrophage tissue with numerous phagosomes in the macrophages; $\times 5400$. Fig. 8. Outer part of the necrotic zone of degenerate macrophages and epitheloid cells Note the abundance of wax-like matter in this tissue; $\times 7000$. Fig. 9 . Inner part of the necrotic zone at the worm's tegument. Note the homogeneous electron-lucent layer between the worm and the wax-like matter; $\times 16900$. AE: amorphous electron-dense material; BL: burst or lysed mitochondrion; CF: collagen fibres; CO: crypt of the outer membrane of the tegument; EL: drop of electron-lucent matter; FB: fibroblast; FC: supporting fibre inside a crypt of the outer membrane; FP: finger-like protuberance of the tegument's outer membrane; HG: heterophilic granulocyte; MF: microfilaments; MI: mitochondrion; MP: macrophage; NU: nucleus; OS: osmiophilic wax-like substance; PA: phagosome (phagolysosome?); RE: rough endoplasmic reticulum; SC: surface coat; SL: striped layer of the tegument 

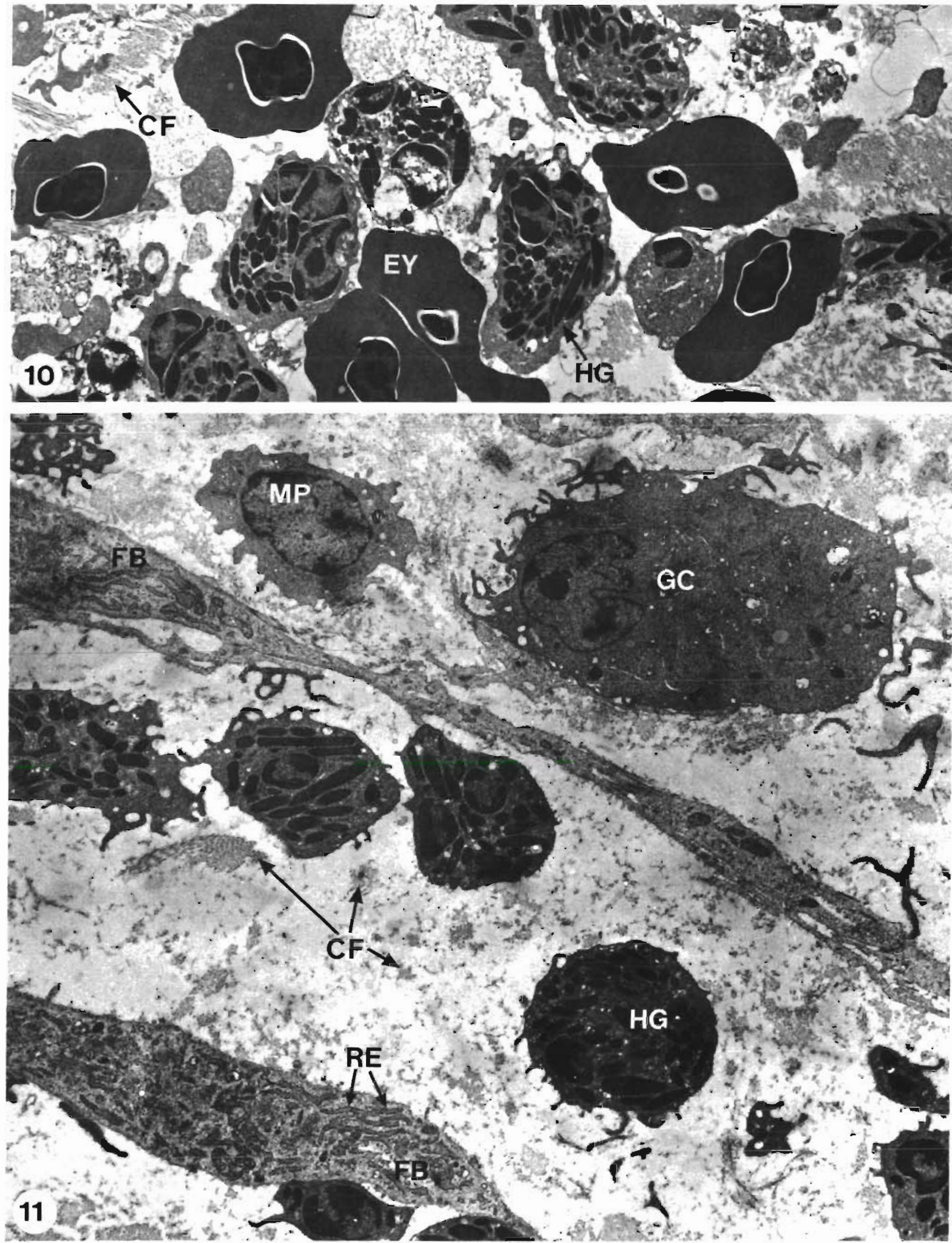


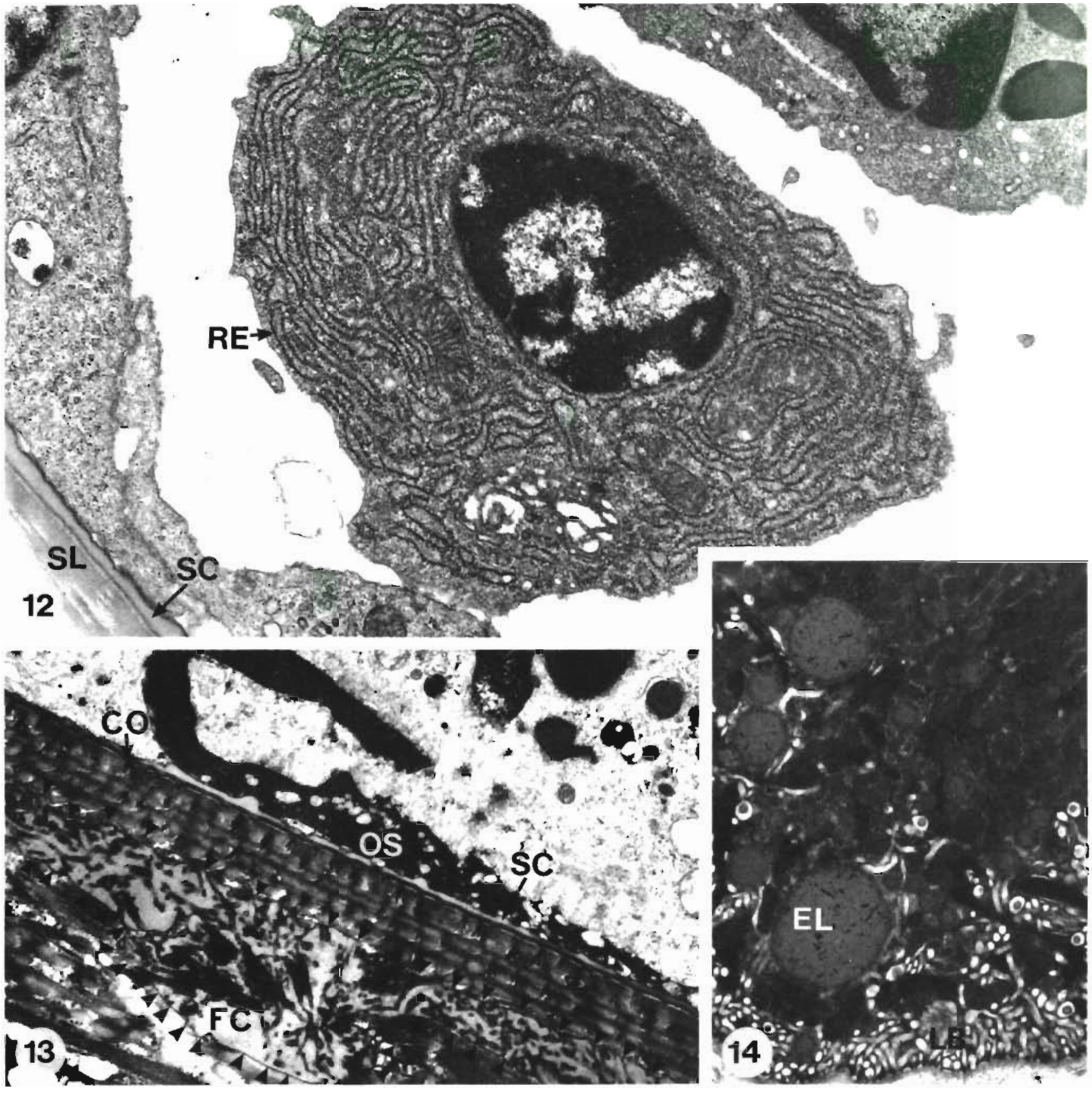

Figs. 12 to 14. Transmission electron micrographs of duck host's defence cells (Fig. 12) and presoma tegument of Filicollis anatis (Figs 13, 14). Fig. 12. Plasma cell. Note the abundance of rough endoplasmic reticulum; $\times 16400$. Fig. 13. Bulbus tegument with fused crypts of the outer membrane. Arrowheads: caverns of fused crypts; $\times 14100$. Fig. 14 . Basal part of the lemniscus; $\times 14100$. CO: crypt of tegument's outer membrane; EL: drop of moderately osmiophilic substance; FC: fused crypts of the outer membrane; LB: labyrinthine basal membrane; OS: osmiophilic wax-like substance; RE: rough endoplasmic reticulum; SC: surface coat; SL: striped layer

Figs. $10 \& 11$. Transmission electron micrographs of the loose mass of connective tissue at the periphery of the capsule around the bulbus of a mature female Filicollis anatis (cf. Fig.4). Fig. 10. Focal haemorrhage in the connective tissue. Note that the erythrocytes have infiltrated the tissue; $\times 5400$. Fig. 11. Non-haemorrhagic area with fibroblasts, macrophages and macrophage giant cells; $\times 5400$. CF: collagen fibres; EY: erythrocyte; FB: fibroblast; GC: macrophage giant cell; HG: heterophilic granulocyte; 
In palaeacanthocephalan and eoacanthocephalan infestations in fish the worm presoma is surrounded by a tissue reaction consisting of different leucocytes (mainly eosinophilic granulocytes), erythrocytes, and depending on the age of the infestation and the depth of worm penetration, also fibroblasts and collagen fibres (Taraschewski 1988b, 1989a, b). Plasma cells, however, seldom occur. In contrast, the tissue around the presoma of archiacanthocephalans in mammals harbours numerous plasma cells outnumbering the eosinophilic granulocytes after the acute phase of infestation (Taraschewski et al. 1989, Zhao et al. 1990). Concerning birds, our knowledge about the processes of defense and immunization against helminths is rather limited (see Dieterlen-Lièvre 1988). Plasma cells seem to be involved in the defence of ducks against $F$. anatis. However they were not as numerous as in the inflammatory tissue of mammals infested with archiacanthocephalans (Taraschewski et al. 1989, Zhao et al. 1990). The capsule around the presoma of $F$. anatis in domestic ducks is characterized by an abundance of heterophilic granulocytes and the presence of macrophage giant cells. This reflects the composition of leucocytes in the avian blood (Dieterlen-Lièvre 1988).

Pflugfelder (1956) stated that Filicollis anatis was conspicuously more pathogenic than the small palaeacanthocephalan Polymorphus minutus, and Hofmann et al. (1989) reported mortality among domestic ducks infested with $F$. anatis. It is not yet clear whether this pathogenicity results from the tissue alterations combined with inflammations and possible secondary infections by microorganisms or from the absorbance of nutrients from the host's intestinal lumen, or from all of these factors.

Acknowledgements. Thanks are due to Prof. Dr R. Ippen (Berlin) and to Dr G. Gräfner (Schwerin) for encouragement of the project by providing their facilities and experience. We also thank Miss C. Brefeld (Bochum) for technical assistance and Prof. Dr H. Mehlhorn (Bochum) for his scientific support.

Responsible Subject Editor: P. Zwart, Utrecht, The Netherlands

\section{LITERATURE CITED}

Dieterlen-Lièvre, F. (1988). Birds. In: Rowley, A. F., Ratcliffe, N. A. (eds.) Vertebrate blood cells. Cambridge University Press, Cambridge, p. 257-336

Hofmann, U., Gräfner, G., Tschemer, W. (1989) Epizootiologie, klinischer Verlauf und Diagnostik der Akanthozephalose (Filikollose) bei Hausenten. Mh. Vet.Med. 44: 576-578

Moore, J., Bell, D. H. (1983). Pathology (?) of Plagiorhynchus cylindraceus in the starling, Sturnus vulgaris. J. Parasitol. 69: $387-390$

Nickol, B. B. (1985). Epizootiologie. In: Crompton, D. W T., Nickol, B. B. (eds.) Biology of the Acanthocephala. Cambridge University Press, Cambridge, p. 307-346

Pflugfelder, $\mathrm{O}$. (1956). Abwehrreaktionen der Wirtstiere von Polymorphus boschadis Schr. (Acanthocephala). Z. ParasitenKde. 17: 371-382

Soliman, K. N. (1955). Observations on some helminth parasites from ducks in Southern England. J. Helminthol. 29: 17-26

Styczynska, E. (1958). Some observations on the development and bionomics of larvae of Filicollis anatis Schrank (Parasitofauna of the biocoenosis of Druzno Lake, Part VII). Acta Parasitol. Pol. 6: 213-224

Taraschewski, H. (1988a). Acanthocephala. In: Mehlhorn, H. (ed.) Parasitology in focus. Springer Verlag, New York, p. $249-254,283-287,344-352,522-529$

Taraschewski, H. (1988b). Host-parasite interface of fish acanthocephalans. 1. Acanthocephalus anguillae (Palaeacanthocephala) in naturally infected fishes: LM and TEM investigations. Dis. aquat. Org. 4: 109-119

Taraschewski, H. (1989a). Acanthocephalus anguillae in intra- and extraintestinal positions in experimentally infected juveniles of goldfish and carp and in sticklebacks. J. Parasitol. 75: 108-118

Taraschewski, H. (1989b). Host-parasite interface of Paratenuisentis ambiguus (Eoacanthocephala) in naturally infected eel and in laboratory-infected sticklebacks and juvenile carp and rainbow trout. J. Parasitol. 75: 911-919

Taraschewski, H., Sagani, C., Mehlhorn, H. (1989). Ultrastructural study of the host-parasite interface of Moniliformis moniliformis (Archiacanthocephala) in laboratory-infected rats. J. Parasitol. 75: 288-296

Zhao, B., Taraschewski, H., Mehlhorn, H. (1990). Licht- und elektronenmikroskopische Untersuchungen zur Histopathogenität von Macracanthorhynchus hirudinaceus (Archiacanthocephala) in experimentell infizierten Hausschweinen. Parasitol. Res. 76: 355-359

Manuscript first received: May 23, 1991

Revised version accepted: August 19, 1991 